\title{
The synthesis of new pyrazolo[1,5-a]pyrimidine derivatives
}

\author{
Ahmad Poursattar Marjani, Jabbar Khalafy, ${ }^{*}$ Fatemeh Salami, and Mahnaz Ezzati \\ Department of Chemistry, Faculty of Science, Urmia University, Urmia 57154, Iran \\ E-mail:jkhalafi@yahoo.com; j.khalafi@urmia.ac.ir
}

DOI: $\underline{\text { http://dx.doi.org/10.3998/ark.5550190.p009.062 }}$

\begin{abstract}
A simple high-yielding procedure for the synthesis of novel pyrazolo[1,5-a]pyrimidine analogues is reported via the condensation of 1,3-diketones or keto ester with substituted 5aminopyrazoles in presence of $\mathrm{H}_{2} \mathrm{SO}_{4}$ using $\mathrm{AcOH}$ as solvent.
\end{abstract}

Keywords: 1 ,3-Diketones, $\beta$-ketoesters, 5-aminopyrazoles, pyrazolo[1,5-a]pyrimidines

\section{Introduction}

The pyrazolopyrimidine derivatives are an important class of heterocyclic compounds with pharmacological and biological activities, such as the antibacterial, ${ }^{1}$ antiviral, ${ }^{2}$ cytotoxic, ${ }^{3}$ antidepressant, ${ }^{4}$ neuroleptic, ${ }^{5}$ tuberculostatic, ${ }^{6}$ antihypertensive, ${ }^{7}$ analgesic ${ }^{8}$ and antimicrobial activity. ${ }^{9}$ The pyrazolo[1,5-a]pyrimidines as bicyclic heterocycles have an important synthetic value in the preparation of drugs with anticancer activities. ${ }^{10-15}$ The most common methods for synthesis of pyrazolo[1,5-a]pyrimidine derivatives are cyclocondensations of 5-aminopyrazoles with bifunctional reagents. ${ }^{16}$ The synthesis of 2 -anilinopyrazolo[1,5-a]pyrimidine derivatives as c-Src kinase inhibitors has been reported. ${ }^{17}$

In continuation of our studies on the synthesis of bi-, tri- and tetracyclic heterocycles, ${ }^{18-25}$ herein we report a convenient method for the synthesis of new pyrazolo[1,5-a]pyrimidine derivatives with possible pharmaceutical applications.

\section{Result and Discussion}

The reaction of 5-amino-3-arylamino-1H-pyrazole-4-carbonitriles (1a-f) and ethyl 5-amino-3arylamino-1H-pyrazole-4-carboxylate (2a,b) with pentane-2,4-dione, ethyl acetoacetate, ethyl isobutyrylacetate and ethyl butyrylacetate (3a-d) afforded the corresponding pyrazolo[1,5-a]pyrimidine derivatives (4a-m) in 87-95\% yield, as shown in Scheme 1 . 


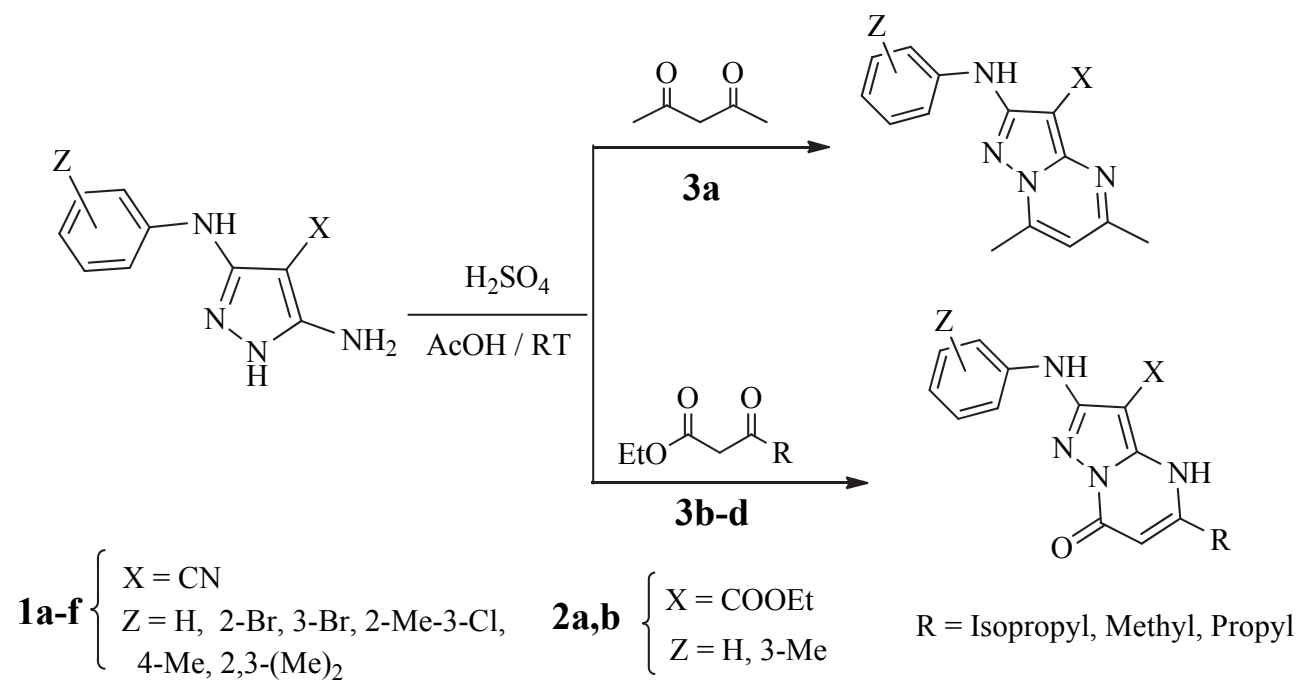

Scheme 1. Synthesis of pyrazolo[1,5-a]pyrimidine derivatives (4a-i, $\mathbf{4 l - m} \mathbf{4 j} \mathbf{j}, \mathbf{k})$.

Thirteen examples of the conversion of 5-amino-3-arylamino- $1 H$-pyrazole-4-carbonitriles (1a-f) and ethyl 5-amino-3-arylamino-1H-pyrazole-4-carboxylate (2a,b) to the corresponding 4,7-dihydropyrazolo[1,5-a]pyrimidine derivatives (4a-m) along with reaction time, melting points and yields are listed in Table 1 .

Table 1. The physical properties, yields and reaction condition for compounds $\mathbf{4 a - m}$

\begin{tabular}{|c|c|c|c|c|c|c|}
\hline Entry & $\begin{array}{c}\text { Pyrazole } \\
\text { derivatives (1a-f) } \\
/(\mathbf{2} \mathbf{a}, \mathbf{b})\end{array}$ & $\begin{array}{l}\text { 1,3-Diketone or } \\
\text { keto ester }(\mathbf{3 a - d})\end{array}$ & Product (4a-m) & $\begin{array}{l}\text { Time } \\
\text { (h) }\end{array}$ & $\begin{array}{l}\text { Yield } \\
(\%)\end{array}$ & $\begin{array}{l}\text { M.p. } \\
\left({ }^{\circ} \mathrm{C}\right)\end{array}$ \\
\hline 1 & $1 \mathbf{a}$ & 3d & $\mathbf{a}$ & 5 & 89 & 297-299 \\
\hline 2 & 1d & 3d & $4 b$ & 7 & 87 & $330-332$ \\
\hline 3 & $1 e$ & 3d & 4 & 8 & 90 & $244-246$ \\
\hline
\end{tabular}


Table 1. Continued

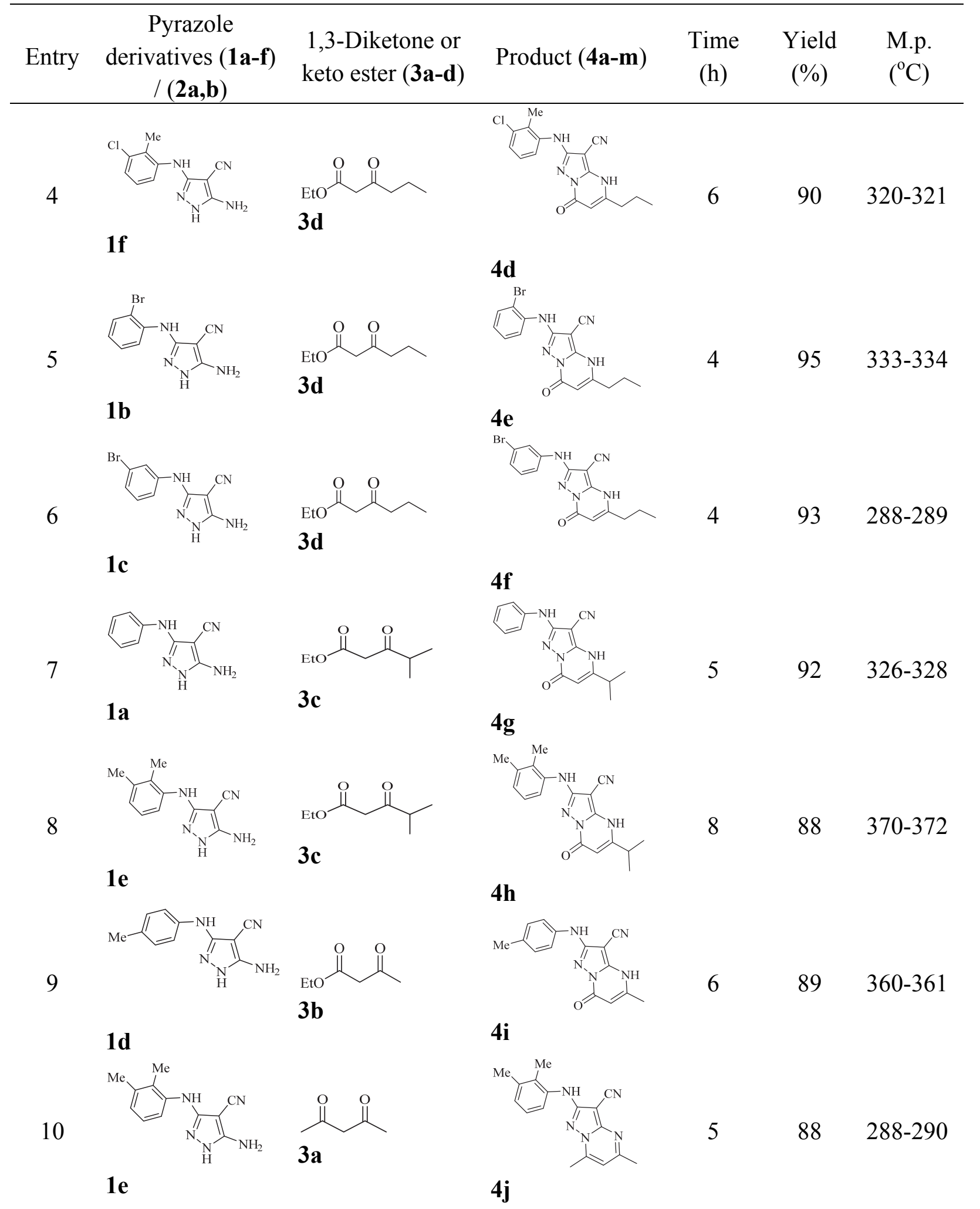


Table 1. Continued

\begin{tabular}{|c|c|c|c|c|c|c|}
\hline Entry & $\begin{array}{c}\text { Pyrazole } \\
\text { derivatives (1a-f) } \\
/(\mathbf{2} \mathbf{a}, \mathbf{b})\end{array}$ & $\begin{array}{l}\text { 1,3-Diketone or } \\
\text { keto ester (3a-d) }\end{array}$ & Product (4a-m) & $\begin{array}{l}\text { Time } \\
\text { (h) }\end{array}$ & $\begin{array}{l}\text { Yield } \\
(\%)\end{array}$ & $\begin{array}{l}\text { M.p. } \\
\left({ }^{\circ} \mathrm{C}\right)\end{array}$ \\
\hline 11 & $2 a$ & $\mathbf{3 a}$ & $4 k$ & 4 & 92 & $159-160$ \\
\hline 12 & $2 \mathbf{b}$ & $3 c$ & 41 & 4.5 & 91 & $205-206$ \\
\hline 13 & $2 b$ & $\begin{array}{l}\mathrm{EtO}^{-} \\
\mathbf{3 d}\end{array}$ & $4 m$ & 4 & 94 & $209-210$ \\
\hline
\end{tabular}

The proposed mechanism for the formation of the fused pyrimidinones may be explained by Scheme 2.

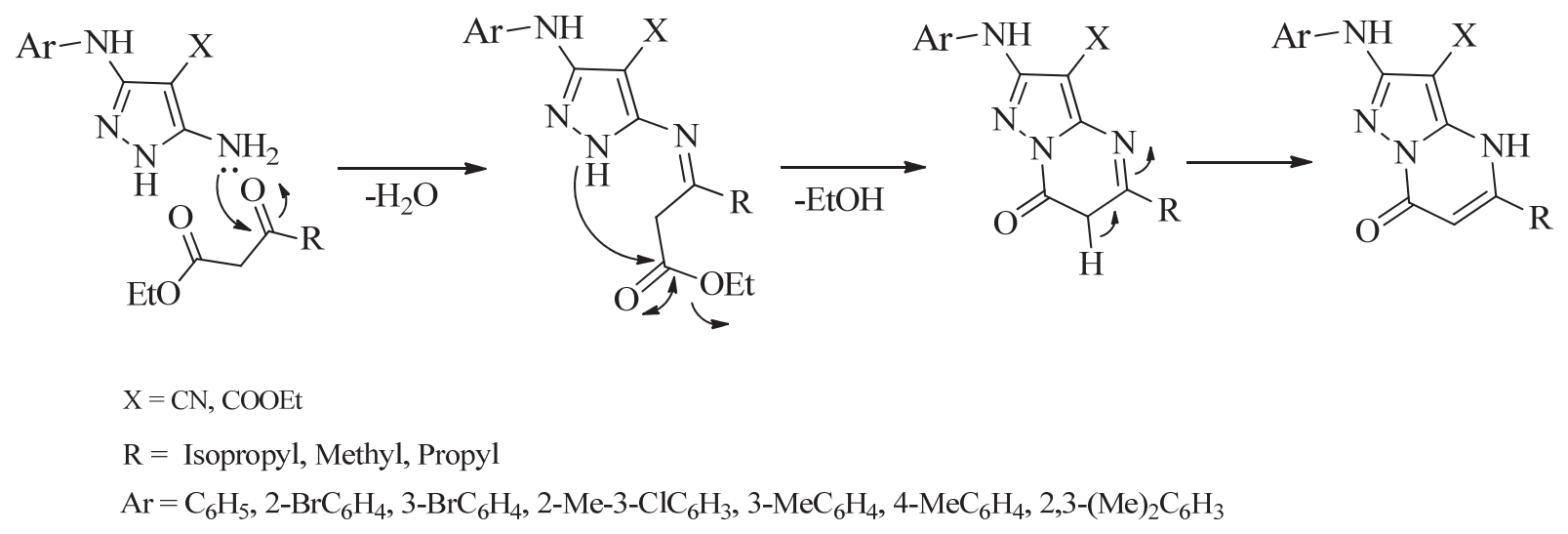

Scheme 2. The proposed mechanism for the formation of compounds (4a-i, $4 \mathbf{l}$ and $\mathbf{4 m})$.

The proposed mechanism for the formation of pyrazolo[1,5-a]pyrimidine derivatives $\mathbf{4} \mathbf{j}, \mathbf{k}$ is shown in Scheme 3. 


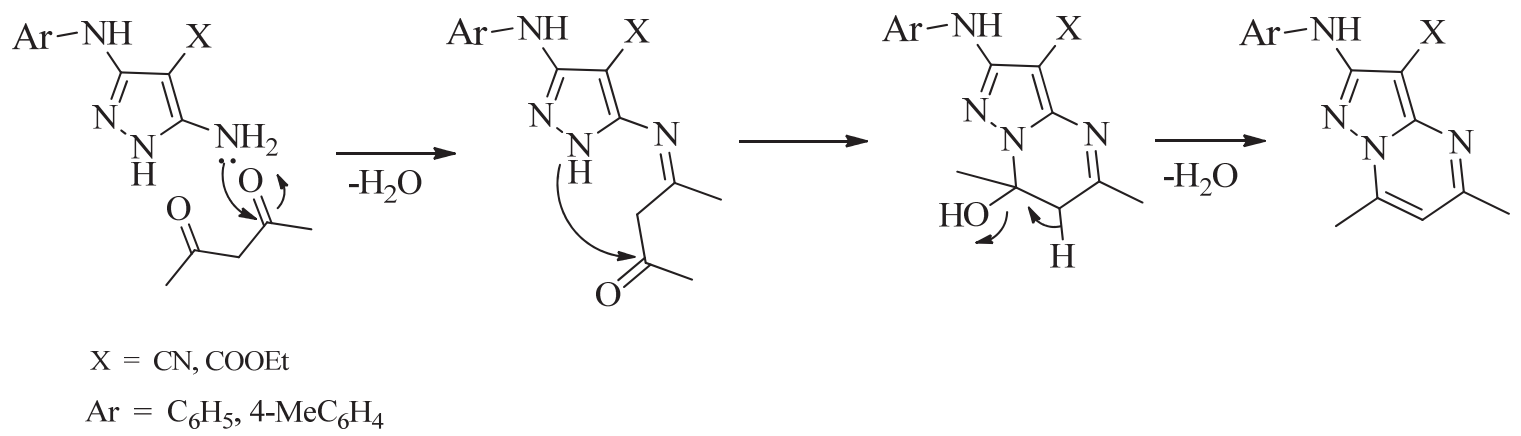

Scheme 3. The proposed reaction mechanism for the formation of compounds $4 \mathbf{j}, \mathbf{k}$.

The structure of all products were confirmed by their ${ }^{1} \mathrm{H}$-NMR, ${ }^{13} \mathrm{C}-\mathrm{NMR}$ and FT-IR spectral data and by elemental analysis.

\section{Experimental Section}

General. The chemicals used in this work were purchased from Acros and Merck companies and were used without purification. Freshly distilled solvents are used throughout; anhydrous solvents are dried according to Perrin and Armarego. ${ }^{26}$ Melting Points were measured on an Electrothermal 9200 apparatus and are uncorrected. FT-IR spectra were recorded via a Thermonicolet (Nexus 670) spectrometer using $\mathrm{KBr}$ discs. ${ }^{1} \mathrm{H}(300 \mathrm{MHz})$ and ${ }^{13} \mathrm{C}(75.5 \mathrm{MHz})$ NMR spectra were recorded on a Bruker DRX-300 Avance spectrometer in DMSO- $d_{6}$ using TMS as the internal reference. Microanalyses are performed on Leco Analyzer 932.

General procedure for the Synthesis of 4,7-dihydropyrazolo[1,5-a]pyrimidine (4a-i, 4l, 4m) and pyrazolo[1,5- $a]$ pyrimidine derivatives $(\mathbf{4 j}, \mathbf{4 k})$. To a solution of pyrazole derivatives ${ }^{27}(1$ mmol) in acetic acid $(20 \mathrm{~mL}), 1,3$-diketones $(2 \mathrm{mmol})$ and one drop of concentrated $\mathrm{H}_{2} \mathrm{SO}_{4}$ was added and then stirred at room temperature until the reaction was completed as monitored by TLC $\left(\mathrm{CHCl}_{3} / \mathrm{MeOH} / \mathrm{CH}_{3} \mathrm{CN}\right.$ v/v, 30: 3: 1). Ice-water $(10 \mathrm{~mL})$ was added to the reaction mixture. The precipitate was filtered, washed with cold water and dried to give the corresponding 4,7dihydropyrazolo[1,5- $a]$ pyrimidine or pyrazolo $[1,5-a]$ pyrimidine derivatives in $87-95 \%$ yields.

7-Oxo-2-phenylamino-5-propyl-4,7-dihydropyrazolo[1,5-a]pyrimidine-3-carbonitrile (4a). White crystals; 89\%; mp 297-299 ${ }^{\circ} \mathrm{C}$; IR $\left(v_{\max }, \mathrm{cm}^{-1}\right): 3314,3156,3061,2959,2817,2231,1670$, $1635,1596,1560,1459,1379,1220,1153,1087,821,754,689,610 .{ }^{1} \mathrm{H}$ NMR $(300 \mathrm{MHz}$, DMSO- $d_{6}$ ): $\delta_{\mathrm{H}} 0.94\left(\mathrm{t}, 3 \mathrm{H}, J 7.2 \mathrm{~Hz}, \mathrm{CH}_{3}\right), 1.67$ (sext, $2 \mathrm{H}, J 7.2 \mathrm{~Hz}, \mathrm{CH}_{2}$ ), 2.54 (t, $2 \mathrm{H}, J 7.2 \mathrm{~Hz}$, $\mathrm{CH}_{2}$ ), 5.80 (s, 1H, CH), 6.93 (t, 1H, J 7.4 Hz, ArH), 7.29 (t, 2H, J 7.4 Hz, ArH), 7.72 (d, 2H, J $8.4 \mathrm{~Hz}, \mathrm{ArH}), 9.16\left(\mathrm{~s}, 1 \mathrm{H}\right.$, exchanged by $\mathrm{D}_{2} \mathrm{O}$ addition, $\left.\mathrm{NH}\right), 12.97\left(\mathrm{~s}, 1 \mathrm{H}\right.$, exchanged by $\mathrm{D}_{2} \mathrm{O}$ addition, NH). ${ }^{13} \mathrm{C}$ NMR $\left(75.5 \mathrm{MHz}\right.$, DMSO- $\left.d_{6}\right): \delta_{\mathrm{c}} 13.74,21.78,34.29,64.95,98.89,112.97$, 
118.11, 121.42, 129.07, 141.41, 145.96, 153.82, 154.20, 155.03; Anal. Calc. for $\mathrm{C}_{16} \mathrm{H}_{15} \mathrm{~N}_{5} \mathrm{O}$ : C 65.52 ; H 5.15; N 23.88. Found: C 65.44; H 5.23; N 23.98\%.

7-Oxo-5-propyl-2-(p-tolylamino)-4,7-dihydropyrazolo[1,5-a]pyrimidine-3-carbonitrile (4b). White crystals; 87\%; mp 330-332 ${ }^{\circ} \mathrm{C}$; IR $\left(v_{\max }, \mathrm{cm}^{-1}\right): 3302,3206,3138,3085,2962,2929,2873$, 2228, 1673, 1614, 1590, 1556, 1524, 1451, 1384, 1293, 811, 501. ${ }^{1} \mathrm{H}$ NMR (300 MHz, DMSO$\left.d_{6}\right): \delta_{\mathrm{H}} 0.92\left(\mathrm{t}, 3 \mathrm{H}, J 7.2 \mathrm{~Hz}, \mathrm{CH}_{3}\right), 1.65\left(\mathrm{sext}, 2 \mathrm{H}, J 7.5 \mathrm{~Hz}, \mathrm{CH}_{2}\right), 2.24\left(\mathrm{~s}, 3 \mathrm{H}, \mathrm{CH}_{3}\right), 2.52(\mathrm{t}, 2 \mathrm{H}$, $\left.J 7.5 \mathrm{~Hz}, \mathrm{CH}_{2}\right), 5.77$ (s, 1H, CH), 7.08 (d, 2H, J 8.1 Hz, ArH), 7.59 (d, 2H, J 8.1 Hz, ArH), 9.02 (s, $1 \mathrm{H}$, exchanged by $\mathrm{D}_{2} \mathrm{O}$ addition, $\left.\mathrm{NH}\right), 12.93\left(\mathrm{~s}, 1 \mathrm{H}\right.$, exchanged by $\mathrm{D}_{2} \mathrm{O}$ addition, $\left.\mathrm{NH}\right) .{ }^{13} \mathrm{C}$ NMR $\left(75.5 \mathrm{MHz}, \mathrm{DMSO}-d_{6}\right): \delta_{\mathrm{c}} 13.75,20.79,21.77,34.21,64.73,98.88,113.03,118.28$, 129.46, 130.19, 138.93, 145.95, 153.97, 154.03, 155.01; Anal. Calc. for $\mathrm{C}_{17} \mathrm{H}_{17} \mathrm{~N}_{5} \mathrm{O}$ : C 66.43; $\mathrm{H}$ 5.58; N 22.79. Found: C 66.58; H 5.41; N 22.67\%.

2-[(2,3-Dimethylphenyl)amino]-7-oxo-5-propyl-4,7-dihydropyrazolo[1,5-a]pyrimidine-3carbonitrile (4c). White crystals; 90\%; mp 244-246 ${ }^{\circ} \mathrm{C}$; IR $\left(v_{\max }, \mathrm{cm}^{-1}\right): 3387,3166,3085,2961$, 2214, 1681, 1629, 1588, 1543, 1512, 1476, 1442, 1385, 1302, 1199, 1093, 764. ${ }^{1} \mathrm{H}$ NMR (300 MHz, DMSO- $d_{6}$ ): $\delta_{\mathrm{H}} 0.90\left(\mathrm{t}, 3 \mathrm{H}, J 6.9 \mathrm{~Hz}, \mathrm{CH}_{3}\right.$ ), 1.61 (sext, 2H, J 6.9 Hz, CH 2 ), 2.09 (s, 3H, $\left.\mathrm{CH}_{3}\right), 2.23\left(\mathrm{~s}, 3 \mathrm{H}, \mathrm{CH}_{3}\right), 2.50\left(\mathrm{t}, 2 \mathrm{H}, J 6.9 \mathrm{~Hz}, \mathrm{CH}_{2}\right), 5.71(\mathrm{~s}, 1 \mathrm{H}, \mathrm{CH}), 6.95(\mathrm{~d}, 1 \mathrm{H}, J 6.6 \mathrm{~Hz}$, ArH), 6.99-7.05 (m, 1H, ArH), 7.16 (bd, 1H, J 7.5 Hz, ArH), 8.37 (s, 1H, exchanged by $\mathrm{D}_{2} \mathrm{O}$ addition, $\mathrm{NH}), 12.90\left(\mathrm{~s}, 1 \mathrm{H}\right.$, exchanged by $\mathrm{D}_{2} \mathrm{O}$ addition, $\left.\mathrm{NH}\right) .{ }^{13} \mathrm{C}$ NMR $(75.5 \mathrm{MHz}, \mathrm{DMSO}-$ $\left.d_{6}\right): \delta_{\mathrm{c}} 13.73,14.40,20.67,21.78,34.17,64.59,98.68,113.04,122.44,125.85,126.52,131.14$, 137.40, 139.05, 146.17, 153.87, 155.06, 156.21; Anal. Calc. for $\mathrm{C}_{18} \mathrm{H}_{19} \mathrm{~N}_{5} \mathrm{O}$ : C 67.27; $\mathrm{H}$ 5.96; $\mathrm{N}$ 21.79. Found: C 67.16; H 6.02; N 21.81\%.

2-[(3-Chloro-2-methylphenyl)amino]-7-oxo-5-propyl-4,7-dihydropyrazolo[1,5-a]pyrimidine-3-carbonitrile (4d). White crystals; 90\%; mp 320-321 ${ }^{\circ} \mathrm{C}$; IR ( $\left.v_{\max }, \mathrm{cm}^{-1}\right): 3446,3170$, 3095, 2971, 2215, 1677, 1634, 1583, 1550, 1461, 1383, 1282, 1218, 1015, 845, 774. ${ }^{1} \mathrm{H}$ NMR (300 MHz, DMSO- $d_{6}$ ): $\delta_{\mathrm{H}} 0.91\left(\mathrm{t}, 3 \mathrm{H}, J 7.2 \mathrm{~Hz}, \mathrm{CH}_{3}\right.$ ), 1.63 (sext, $2 \mathrm{H}, J 7.5 \mathrm{~Hz}, \mathrm{CH}_{2}$ ), 2.23 (s, $\left.3 \mathrm{H}, \mathrm{CH}_{3}\right), 2.53$ (t, 2H, J $\left.7.5 \mathrm{~Hz}, \mathrm{CH}_{2}\right), 5.74(\mathrm{~s}, 1 \mathrm{H}, \mathrm{CH}), 7.14-7.21(\mathrm{~m}, 2 \mathrm{H}, \mathrm{ArH}), 7.34(\mathrm{~d}, 1 \mathrm{H}, J$ $6.9 \mathrm{~Hz}, \mathrm{ArH}), 8.64\left(\mathrm{~s}, 1 \mathrm{H}\right.$, exchanged by $\mathrm{D}_{2} \mathrm{O}$ addition, $\left.\mathrm{NH}\right), 12.96\left(\mathrm{~s}, 1 \mathrm{H}\right.$, exchanged by $\mathrm{D}_{2} \mathrm{O}$ addition, NH). ${ }^{13} \mathrm{C}$ NMR (75.5 MHz, DMSO- $\left.d_{6}\right): \delta_{\mathrm{c}} 13.73,15.53,21.79,34.20,65.08,98.77$, 112.93, 122.77, 125.20, 127.48, 129.97, 134.33, 141.08, 146.13, 154.10, 155.03, 155.53; Anal. Calc. for $\mathrm{C}_{17} \mathrm{H}_{16} \mathrm{ClN}_{5} \mathrm{O}$ : C 59.74; $\mathrm{H} 4.72 ; \mathrm{N} 20.49$. Found: C 59.85; H 4.62; N 20.33\%.

2-[(2-Bromophenyl)amino]-7-oxo-5-propyl-4,7-dihydropyrazolo[1,5-a]pyrimidine-3-carbonitrile (4e). White crystals; 95\%; mp 333-334 ${ }^{\circ} \mathrm{C}$; IR $\left(v_{\max }, \mathrm{cm}^{-1}\right): 3388,3075,2958,288,2216$, $1676,1628,1590,1551,1450,1386,1295,742 .{ }^{1} \mathrm{H}$ NMR $\left(300 \mathrm{MHz}, \mathrm{DMSO}-d_{6}\right): \delta_{\mathrm{H}} 0.91(\mathrm{t}, 3 \mathrm{H}$, $\left.J 6.9 \mathrm{~Hz}, \mathrm{CH}_{3}\right), 1.63$ (sext, 2H, J $\left.6.9 \mathrm{~Hz}, \mathrm{CH}_{2}\right), 2.51$ (t, $\left.2 \mathrm{H}, J 6.9 \mathrm{~Hz}, \mathrm{CH}_{2}\right), 5.77(\mathrm{~s}, 1 \mathrm{H}, \mathrm{CH})$, 7.00-7.04 (m, 1H, ArH), 7.34-39 (m, 1H, ArH), 7.62 (bd, 1H, J 7.8 Hz, ArH), 7.83 (d, 1H, J 7.8 $\mathrm{Hz}, \mathrm{ArH}), 8.19\left(\mathrm{~s}, 1 \mathrm{H}\right.$, exchanged by $\mathrm{D}_{2} \mathrm{O}$ addition, $\left.\mathrm{NH}\right), 13.01\left(\mathrm{~s}, 1 \mathrm{H}\right.$, exchanged by $\mathrm{D}_{2} \mathrm{O}$ addition, NH). ${ }^{13} \mathrm{C}$ NMR (75.5 MHz, DMSO- $\left.d_{6}\right): \delta_{\mathrm{c}} 13.73,21.74,34.25,65.66,98.88,112.69$, $116.07,123.32,125.21,128.85,133.17,138.71,145.68,154.27,154.57,155.04$; Anal. Calc. for $\mathrm{C}_{16} \mathrm{H}_{14} \mathrm{BrN}_{5} \mathrm{O}$ : C 51.63; H 3.79; N 18.82. Found: C 51.51; H 3.85; N 18.94\%. 
2-[(3-Bromophenyl)amino]-7-oxo-5-propyl-4,7-dihydropyrazolo[1,5-a]pyrimidine-3-carbonitrile (4f). White crystals; 93\%; mp 288-289 ${ }^{\circ} \mathrm{C}$; IR $\left(v_{\max }, \mathrm{cm}^{-1}\right): 3325,3071,2966,2221,1673$, $1634,1546,1451,1381,1221,1088,875,677,553 .{ }^{1} \mathrm{H}$ NMR $\left(300 \mathrm{MHz}, \mathrm{DMSO}-d_{6}\right): \delta_{\mathrm{H}} 0.92(\mathrm{t}$, $3 \mathrm{H}, J 7.2 \mathrm{~Hz}, \mathrm{CH}_{3}$ ), 1.64 (sext, 2H, J 7.2 Hz, CH ), 2.52 (t, 2H, J 7.5 Hz, $\mathrm{CH}_{2}$ ), 5.80 (s, 1H, CH), 7.08 (d, 1H, J 7.8 Hz, ArH), 7.24 (t, 1H, J 8.1 Hz, ArH), 7.67 (d, 1H, J 8.1 Hz, ArH), 8.0 (s, 1H, $\mathrm{ArH}), 9.40\left(\mathrm{~s}, 1 \mathrm{H}\right.$, exchanged by $\mathrm{D}_{2} \mathrm{O}$ addition, $\left.\mathrm{NH}\right), 13.03\left(\mathrm{~s}, 1 \mathrm{H}\right.$, exchanged by $\mathrm{D}_{2} \mathrm{O}$ addition, $\mathrm{NH}) .{ }^{13} \mathrm{C}$ NMR $\left(75.5 \mathrm{MHz}, \mathrm{DMSO}-d_{6}\right): \delta_{\mathrm{c}} 13.74,21.77,34.22,65.15,98.97,112.77,116.82$, $120.10,122.11,123.85,131.04,143.01,145.94,153.30,154.33,154.98$; Anal. Calc. for $\mathrm{C}_{16} \mathrm{H}_{14} \mathrm{BrN}_{5} \mathrm{O}$ : C 51.63; H 3.79; N 18.82. Found: C 51.79; H 3.68; N 18.73\%.

5-Isopropyl-7-oxo-2-phenylamino-4,7-dihydropyrazolo[1,5-a]pyrimidine-3-carbonitrile (4g). ${ }^{28}$ White crystals; 92\%, mp 326-328 ${ }^{\circ} \mathrm{C}$; IR $\left(v_{\max }, \mathrm{cm}^{-1}\right): 3395,3152,3060,2976,2825$, 2219, 1672, 1629, 1592, 1551, 1498, 1460, 1392, 1314, 1234, 1173, 1080, 842, 754, 692, 560, 522, 469. ${ }^{1} \mathrm{H}$ NMR (300 MHz, DMSO-d $\left.)_{6}\right): \delta_{\mathrm{H}} 1.22\left(\mathrm{~d}, 6 \mathrm{H}, J 6.9 \mathrm{~Hz}, 2 \times \mathrm{CH}_{3}\right), 2.86(\mathrm{sep}, 1 \mathrm{H}, J 6.9$ $\mathrm{Hz}, \mathrm{CH}), 5.84$ (s, 1H, CH), 6.90 (t, 1H, J 7.2 Hz, ArH), 7.29-7.34 (m, 2H, J 7.8 Hz, ArH), 7.72 (d, 2H, J 7.5 Hz, ArH), 8.31 (s, 1H, exchanged by $\mathrm{D}_{2} \mathrm{O}$ addition, $\left.\mathrm{NH}\right), 12.90(\mathrm{~s}, 1 \mathrm{H}$, exchanged by $\mathrm{D}_{2} \mathrm{O}$ addition, $\left.\mathrm{NH}\right) .{ }^{13} \mathrm{C} \mathrm{NMR}\left(75.5 \mathrm{MHz}, \mathrm{DMSO}-d_{6}\right): \delta_{\mathrm{c}} 21.79,31.90,64.93,96.71,113.39$, $117.84,121.53,129.33,140.64,143.38,153.17,155.44,159.89$; Anal. Calc. for $\mathrm{C}_{16} \mathrm{H}_{15} \mathrm{~N}_{5} \mathrm{O}$ : C 65.52; H 5.15; N 23.88. Found: C 65.66; H 5.01; N 23.75\%.

2-[(2,3-Dimethylphenyl)amino]-5-isopropyl-7-oxo-4,7-dihydropyrazolo[1,5-a]pyrimidine-3carbonitrile (4h). White crystals; 88\%; mp 370-372 ${ }^{\circ} \mathrm{C}$; IR $\left(v_{\max }, \mathrm{cm}^{-1}\right): 3376,3168,3085,2970$, 2216, 1682, 1629, 1587, 1536, 1513, 1468, 1388, 1323, 1228, 1172, 1120, 1083, 918, 825, 768. ${ }^{1} \mathrm{H}$ NMR (300 MHz, DMSO-d $): \delta_{\mathrm{H}} 1.21\left(\mathrm{~d}, 6 \mathrm{H}, J 6.9 \mathrm{~Hz}, 2 \times \mathrm{CH}_{3}\right), 2.09\left(\mathrm{~s}, 3 \mathrm{H}, \mathrm{CH}_{3}\right), 2.29(\mathrm{~s}$, $\left.3 \mathrm{H}, \mathrm{CH}_{3}\right), 2.84(\mathrm{sep}, 1 \mathrm{H}, J 6.9 \mathrm{~Hz}, \mathrm{CH}), 5.71(\mathrm{~s}, 1 \mathrm{H}, \mathrm{CH}), 6.93(\mathrm{~d}, 1 \mathrm{H}, J 7.2 \mathrm{~Hz}, \mathrm{ArH}), 7.01(\mathrm{t}$, $1 \mathrm{H}, J 7.5 \mathrm{~Hz}, \mathrm{ArH}), 7.16$ (d, 1H, J 7.5 Hz, ArH), 8.35 (s, 1H, exchanged by $\mathrm{D}_{2} \mathrm{O}$ addition, $\mathrm{NH}$ ), $12.82\left(\mathrm{~s}, 1 \mathrm{H}\right.$, exchanged by $\mathrm{D}_{2} \mathrm{O}$ addition, $\left.\mathrm{NH}\right) .{ }^{13} \mathrm{C} \mathrm{NMR}\left(75.5 \mathrm{MHz}, \mathrm{DMSO}-d_{6}\right): \delta_{\mathrm{c}} 14.39$, 20.65, 21.42, 31.45, 64.70, 95.95, 113.06, 122.47, 125.82, 126.52, 131.15, 137.39, 139.03, 145.89, 155.40, 156.38, 159.43; Anal. Calc. for $\mathrm{C}_{18} \mathrm{H}_{19} \mathrm{~N}_{5} \mathrm{O}$ : C 67.27; H 5.96; N 21.79. Found: C 67.11 ; H 6.08; N 21.66\%.

5-Methyl-7-oxo-2-(p-tolylamino)-4,7-dihydropyrazolo[1,5-a]pyrimidine-3-carbonitrile (4i). White crystals; 89\%; mp 360-361 ${ }^{\circ} \mathrm{C}$; IR $\left(v_{\max }, \mathrm{cm}^{-1}\right): 3384,3167,3077,2975,2833,2216,1678$, 1634, 1591, 1550, 1450, 1396, 1308, 1221, 1169, 1021, 840, 739, 663, 562, 472. ${ }^{1} \mathrm{H}$ NMR (300 MHz, DMSO- $\left.d_{6}\right): \delta_{\mathrm{H}} 2.23\left(\mathrm{~s}, 3 \mathrm{H}, \mathrm{CH}_{3}\right), 2.27\left(\mathrm{~s}, 3 \mathrm{H}, \mathrm{CH}_{3}\right), 5.74(\mathrm{~s}, 1 \mathrm{H}, \mathrm{CH}), 7.07$ (d, 2H, J 8.1 $\mathrm{Hz}, \mathrm{ArH}), 7.59$ (d, 2H, J 8.1 Hz, ArH), 9.04 (s, 1H, exchanged by $\mathrm{D}_{2} \mathrm{O}$ addition, $\left.\mathrm{NH}\right), 13.01$ (s, $1 \mathrm{H}$, exchanged by $\mathrm{D}_{2} \mathrm{O}$ addition, $\left.\mathrm{NH}\right) .{ }^{13} \mathrm{C} \mathrm{NMR}\left(75.5 \mathrm{MHz}, \mathrm{DMSO}-d_{6}\right): \delta_{\mathrm{c}} 18.53,20.79,64.55$, 99.52, 113.00, 118.24, 129.47, 130.18, 138.91, 145.84, 150.50, 153.86, 154.91; Anal. Calc. for $\mathrm{C}_{15} \mathrm{H}_{13} \mathrm{~N}_{5} \mathrm{O}$ : C 64.51; H 4.69; N 25.07. Found: C 64.40; H 4.78; N 25.19\%.

2-[(2,3-Dimethylphenyl)amino]-5,7-dimethylpyrazolo[1,5-a]pyrimidine-3-carbonitrile (4j). White crystals; 88\%; mp 288-290 ${ }^{\circ} \mathrm{C}$; IR $\left(v_{\max }, \mathrm{cm}^{-1}\right): 3436,3054,2995,2917,2208,1609,1591$, 1486, 1274, 1187, 1062, 778, 438. ${ }^{1} \mathrm{H}$ NMR (300 MHz, DMSO- $\left.d_{6}\right): \delta_{\mathrm{H}} 2.13\left(\mathrm{~s}, 3 \mathrm{H}, \mathrm{CH}_{3}\right), 2.26$ (s, 3H, $\left.\mathrm{CH}_{3}\right), 2.49$ (s, 3H, $\left.\mathrm{CH}_{3}\right), 2.53\left(\mathrm{~s}, 3 \mathrm{H}, \mathrm{CH}_{3}\right), 6.94(\mathrm{~s}, 1 \mathrm{H}, \mathrm{ArH}), 6.98(\mathrm{~d}, 1 \mathrm{H}, J \mathrm{~J} .2 \mathrm{~Hz}$, 
ArH), 7.05 (t, 1H, J 7.5 Hz, ArH), 7.21 (d, 1H, J 7.8 Hz, ArH), 8.74 (s, 1H, exchanged by $\mathrm{D}_{2} \mathrm{O}$ addition, NH). ${ }^{13} \mathrm{C}$ NMR (75.5 MHz, DMSO- $\left.d_{6}\right): \delta_{\mathrm{c}} 14.57,17.02,20.70,24.40,66.90,110.40$, 114.20, 122.68, 125.86, 126.84, 131.52, 137.54, 138.59, 146.41, 151.41, 158.69, 161.66; Anal. Calc. for $\mathrm{C}_{17} \mathrm{H}_{17} \mathrm{~N}_{5}$ : C 70.08; H 5.88; N 24.04. Found: C 69.97; H 5.76; N 24.20\%.

Ethyl 5,7-dimethyl-2-(phenylamino)pyrazolo[1,5-a]pyrimidine-3-carboxylate (4k). White crystals; 92\%; mp 159-160 ${ }^{\circ} \mathrm{C}$; IR $\left(v_{\max }, \mathrm{cm}^{-1}\right): 3319,2971,2927,1656,1597,1563,1497,1428$, $1388,1295,1238,1203,1154,1099,1028,892,791,691 .{ }^{1} \mathrm{H}$ NMR $\left(300 \mathrm{MHz}, \mathrm{DMSO}-d_{6}\right): \delta_{\mathrm{H}}$ 1.33 (t, 3H, J $\left.7.2 \mathrm{~Hz}, \mathrm{CH}_{3}\right), 2.46\left(\mathrm{~s}, 3 \mathrm{H}, \mathrm{CH}_{3}\right), 2.60\left(\mathrm{~s}, 3 \mathrm{H}, \mathrm{CH}_{3}\right), 4.31\left(\mathrm{q}, 2 \mathrm{H}, J 7.2 \mathrm{~Hz}, \mathrm{CH}_{2}\right)$, $6.87(\mathrm{~s}, 1 \mathrm{H}, \mathrm{ArH}), 6.95$ (t, 1H, J 7.2 Hz, ArH), 7.32 (t, 2H, J 7.2 Hz, ArH), 7.70 (d, 2H, J 7.8 Hz, $\mathrm{ArH}), 8.97$ (s, $1 \mathrm{H}$, exchanged by $\mathrm{D}_{2} \mathrm{O}$ addition, $\left.\mathrm{NH}\right) .{ }^{13} \mathrm{C}$ NMR $\left(75.5 \mathrm{MHz}, \mathrm{DMSO}-d_{6}\right): \delta_{\mathrm{c}} 14.92$, $17.17,24.85,59.95,110.28,117.86,121.57,129.44,140.56,146.22,147.27,156.92,162.01$, 165.05; Anal. Calc. for $\mathrm{C}_{17} \mathrm{H}_{18} \mathrm{~N}_{4} \mathrm{O}_{2}$ : C 65.79; $\mathrm{H}$ 5.85; $\mathrm{N}$ 18.05. Found: $\mathrm{C}$ 65.83; H 5.76; $\mathrm{N}$ $17.88 \%$.

Ethyl 5-isopropyl-7-oxo-2-(m-tolylamino)-4,7-dihydropyrazolo[1,5-a]pyrimidine-3-carboxylate (4I). White crystals; 91\%; mp 205-206 ${ }^{\circ} \mathrm{C}$; IR ( $\left.v_{\max }, \mathrm{cm}^{-1}\right): 3338,3193,3097,2964,2878$, 1691, 1616, 1599, 1560, 1487, 1460, 1401, 1259, 1155, 1101, 1017, 885, 781, 690, 611, 585. ${ }^{1} \mathrm{H}$ NMR (300 MHz, DMSO- $\left.d_{6}\right): \delta_{\mathrm{H}} 1.23\left(\mathrm{~d}, 6 \mathrm{H}, J 6.9 \mathrm{~Hz}, 2 \times \mathrm{CH}_{3}\right), 1.37\left(\mathrm{t}, 3 \mathrm{H}, J 6.9 \mathrm{~Hz}, \mathrm{CH}_{3}\right), 2.29$ $\left(\mathrm{s}, 3 \mathrm{H}, \mathrm{CH}_{3}\right), 3.20(\mathrm{sep}, 1 \mathrm{H}, J 6.6 \mathrm{~Hz}, \mathrm{CH}), 4.39$ (q, 2H, J $\left.6.9 \mathrm{~Hz}, \mathrm{CH}_{2}\right), 5.83(\mathrm{~s}, 1 \mathrm{H}, \mathrm{CH}), 6.77$ (d, 1H, J $7.5 \mathrm{~Hz}, \mathrm{ArH}), 7.20$ (t, 1H, J 7.8 Hz, ArH), 7.44 (s, 1H, ArH), 7.61 (d, 1H, J 7.8 Hz, $\mathrm{ArH}), 8.23$ (s, $1 \mathrm{H}$, exchanged by $\mathrm{D}_{2} \mathrm{O}$ addition, $\left.\mathrm{NH}\right), 10.99\left(\mathrm{~s}, 1 \mathrm{H}\right.$, exchanged by $\mathrm{D}_{2} \mathrm{O}$ addition, $\mathrm{NH}) .{ }^{13} \mathrm{C}$ NMR $\left(75.5 \mathrm{MHz}, \mathrm{DMSO}-d_{6}\right): \delta_{\mathrm{c}} 14.89,21.74,21.80,30.80,60.63,84.42,96.75$, 115.03, 118.31, 122.32, 129.21, 138.51, 140.59, 143.41, 153.14, 155.36, 159.77, 163.17; Anal. Calc. for $\mathrm{C}_{19} \mathrm{H}_{22} \mathrm{~N}_{4} \mathrm{O}_{3}$ : C 64.39; H 6.26; N 15.81. Found: C 64.42; H 6.17; N 15.68\%.

Ethyl 7-oxo-5-propyl-2-(m-tolylamino)-4,7-dihydropyrazolo[1,5-a]pyrimidine-3-carboxylate $(\mathbf{4 m})$. White crystals; $94 \%$; mp 209-210 ${ }^{\circ} \mathrm{C}$; IR $\left(v_{\max }, \mathrm{cm}^{-1}\right): 3394,3060,2954,1699,1668$, $1602,1563,1498,1444,1355,1276,1557,1122,1025,855,785,745,690,647,523 ;{ }^{1} \mathrm{H} \mathrm{NMR}$ (300 MHz, DMSO- $d_{6}$ ): $\delta_{\mathrm{H}} 0.97\left(\mathrm{t}, 3 \mathrm{H}, J 6.6 \mathrm{~Hz}, \mathrm{CH}_{3}\right), 1.35\left(\mathrm{t}, 3 \mathrm{H}, J 6.9 \mathrm{~Hz}, \mathrm{CH}_{3}\right), 1.63(\mathrm{tq}, 2 \mathrm{H}$, $\left.J 6.6 \mathrm{~Hz}, \mathrm{CH}_{2}\right), 2.29\left(\mathrm{~s}, 3 \mathrm{H}, \mathrm{CH}_{3}\right), 2.65\left(\mathrm{t}, 2 \mathrm{H}, J 6.6 \mathrm{~Hz}, \mathrm{CH}_{2}\right), 4.38\left(\mathrm{q}, 2 \mathrm{H}, J 6.9 \mathrm{~Hz}, \mathrm{CH}_{2}\right), 5.80$ (s, 1H, CH), 6.76 (bd, 1H, J 6.6 Hz, ArH), 7.19 (bt, 1H, J 6.9 Hz, ArH), 7.42 (bs, 1H, ArH), 7.60 (bd, $1 \mathrm{H}, J 7.2 \mathrm{~Hz}, \mathrm{ArH}), 8.23\left(\mathrm{~s}, 1 \mathrm{H}\right.$, exchanged by $\mathrm{D}_{2} \mathrm{O}$ addition, $\left.\mathrm{NH}\right), 11.20(\mathrm{~s}, 1 \mathrm{H}$, exchanged by $\mathrm{D}_{2} \mathrm{O}$ addition, $\left.\mathrm{NH}\right) .{ }^{13} \mathrm{C} \mathrm{NMR}\left(75.5 \mathrm{MHz}, \mathrm{DMSO}-d_{6}\right): \delta_{\mathrm{c}} 13.75,14.90,21.71,21.88,34.11$, $60.61,84.41,99.48,114.99,118.27,122.31,129.20,138.52,140.57,143.52,153.09,154.19$, 155.11, 163.17; Anal. Calc. for $\mathrm{C}_{19} \mathrm{H}_{22} \mathrm{~N}_{4} \mathrm{O}_{3}$ : C 64.39; H 6.26; N 15.81. Found: C 64.48; H 6.03; N $15.72 \%$.

\section{Acknowledgements}

The authors are grateful to Urmia University for financial support. 


\section{Supplementary Information}

${ }^{1} \mathrm{H}$-NMR, ${ }^{13} \mathrm{C}$-NMR and FT-IR spectral data for compounds $\mathbf{4 a - m}$ are available as supplementary information.

\section{References}

1. Bayomi, S. M.; Amin, K. M.; Al-obaid, A. M.; Hares, N. G. Egypt J. Pharm. Sci. 1993, 34, 117.

2. Holla, B. S.; Kalluraya, B.; Sridhar, K. R.; Drake, E.; Thomas, L. M.; Bhandary, K. K.; Levine, M. J. Eur. J. Med. Chem. 1994, 29, 301. http://dx.doi.org/10.1016/0223-5234(94)90100-7

3. Panisheva, E. R.; Kaplina, N. V.; Nikolaeva, I. S.; Phuskina, T. V.; Pershin Khim-Farm, Z. H. Chem. Abst. 1981, 15, 40.

4. Abdou, I. M.; Saleh, A. M.; Zohdi, H. F. Molecule. 2004, 9, 109. http://dx.doi.org/10.3390/90300109

5. Filler, R. Chem Technol. 1974, 4, 752.

http://dx.doi.org/10.3390/90300109

6. Moustafa, M. G.; Zeinab, H. I.; Soad, A. A. Heteroatom Chem. 2004, 1, 57. http://dx.doi.org/10.1002/hc.10212

7. EI-Feky, A.; Abd-Ei-Samii, Z. K. Pharmazie 1996, 51, 540.

8. Vijaya Raj, K. K.; Narayan, B. V.; Ashalatha, N.; Suchita, K. J. Pharmacol. Toxicol. 2006, 1,559 .

9. Amin, M. A.; Iamil, M. M.; Addel, G. A.; Bauomy, A. H.; Ahmad, M. Alexandria J. Pharm. Sci. 2003, 17, 1 .

10. Lane, M. E.; Yu, B.; Rice, A.; Lipson, K. E.; Liang, C.; Sun, L.; Tang, C.; McMahon, G.; Pestell, R. G.; Wadler, S. Cancer Res. 2001, 61, 6170.

http://cancerres.aacrjournals.org/content/61/16/6170

11. Fraley, M. E.; Hoffman, W. F.; Rubino, R. S.; Hungate, R. W.; Tebben, A. J.; Rutledge, R. Z.; McFall, R. C.; Huckle, W. R.; Kendall, R. L.; Coll, K. E.; Thomas, K. A. Bioorg. Med. Chem. Lett. 2002, 12, 2767. http://dx.doi.org/10.1016/S0960-894X(02)00525-5

12. Fraley, M. E.; Rubino, R. S.; Hoffman, W. F.; Hambaugh, S. R.; Arrington, K. L.; Hungate, R. W.; Bilodeau, M. T.; Tebben, A. J.; Rutledge, R. Z.; Kendall, R. L.; McFall, R. C.; Huckle, W. R.; Coll, K. E.; Thomas, K. A. Bioorg. Med. Chem. Lett. 2002, 12, 3537. http://dx.doi.org/10.1016/S0960-894X(02)00827-2

13. Wu, Z.; Fraley, M. E.; Bilodeau, M. T.; Kaufman, M. L.; Tasber, E. S.; Balitza, A. E.; Hartman, G. D.; Coll, K. E.; Rickert, K.; Shipman, J.; Shi, B.; Sepp-Lorenzino, L.; Thomas, K. A. Bioorg. Med. Chem. Lett. 2004, 14, 909. http://dx.doi.org/10.1016/j.bmcl.2003.12.007 
14. Arora, A.; Scholar, E. M. J. Pharmacol. Exp. Ther. 2005, 315, 971.

15. Meijer, L.; Raymond, A. E. Acc. Chem. Res. 2003, 36, 417. http://dx.doi.org/10.1021/ar0201198

16. Senga, K.; Novinson, T.; Wilson, H. R. J. Med. Chem. 1981, 24, 610. http://dx.doi.org/272 10.1021/jm00137a023

17. Mukaiyama, H.; Nishimura, T.; Shiohara, H.; Kobayashi, S.; Komatsu, Y.; Kikuchi, S.; Tsuji, E.; Kamada, N.; Ohnota, H.; Kusama, H. Chem. Pharm. Bull. 2007, 55, 881. http://dx.doi.org/10.1002/chin.200748148

18. Molla Ebrahimlo, A. R.; Khalafy, J.; Prager, R. H. Aust. J. Chem. 2009, 62, 126. http://dx.doi.org/10.1071/CH08370

19. Molla Ebrahimlo, A. R.; Khalafy, J.; Poursattar Marjani, A.; Prager, R. H. Arkivoc 2009, xii, 17. http://dx.doi.org/10.3998/ark.5550190.0010.c03

20. Poursattar Marjani, A.; Khalafy, J.; Chem. Heterocycl. Comp. 2011, 47, 96. http://dx.doi.org/10.1007/s10593-011-0725-0

21. Poursattar Marjani, A.; Khalafy, J.; Molla Ebrahimlo, A. R. Synth. Commun. 2011, 41, 2475. http://dx.doi.org/10.1080/00397911.2010.505701

22. Poursattar Marjani, A.; Khalafy, J.; Molla Ebrahimlo, A. R.; Prager, R. H. Bull. Korean. Chem. Soc. 2011, 32, 2183. http://dx.doi.org/10.5012/bkcs.2011.32.7.2183

23. Poursattar Marjani, A.; Khalafy, J.; Prager, R. H. Chem. Heterocycl. Comp. 2012, 48, 931. http://dx.doi.org/10.1007/s10593-012-1079-y

24. Khalafy, J.; Ezzati, M.; Rimaz, M.; Poursattar Marjani, A.; Yaghoobnejad Asl, H. J. Iran. Chem. Soc. 2014, 11, 1067. http://dx.doi.org/10.1007/s13738-013-0378-2

25. Khalafy, J.; Etivand, N.; Dilmaghani, S.; Ezzati, M.; Poursattar Marjani, A. Tetrahedron Lett. 2014, 55, 3781 . http://dx.doi.org/10.1016/j.tetlet.2014.05.073

26. Perrin, D. D.; Armarego, W. L. F.; In Purification of Laboratory Chemicals, Pergamon Press: Oxford, U.K., 1988.

27. Khalafy, J.; Poursattar Marjani, A.; Salami, F. Tetrahedron Lett. 2014, 55, 6671. http://dx.doi.org/10.1016/j.tetlet.2014.10.061

28. Iwao, T.; Yasuo, S.; Yasunori, T.; Koji, N.; Hiroyuki: T.; JP. Patent 2005-162726, 2005 (CAS No. 824397-75-3). 